\title{
Roles of TRIM32 in Corneal Epithelial Cells After Infection with Herpes Simplex Virus
}

\author{
Hao Cuja,b Ying Liu ${ }^{a, c}$ Yifei Huang ${ }^{a}$ \\ a Department of Ophthalmology, Chinese PLA General Hospital, Beijing, ${ }^{b}$ Department of \\ Ophthalmology, Harbin 242 Hospital, Harbin, Heilongjiang, ' Department of Ophthalmology, The First \\ Affiliated Hospital of Liaoning Medical University, Jinzhou, Liaoning, China
}

\section{Key Words}

Trim32 • Corneal epithelial cells • Herpes Simplex Virus • In vivo • In vitro

\begin{abstract}
Background: Epithelial cells play important roles as a critical barrier in protecting the cornea from microbial pathogens infection. Methods: In this study, we were aiming to investigate the role of E3 ubiquitin ligase tripartite motif protein 32 (TRIM32) in corneal epithelial cells in response to Herpes Simplex Virus type 1 (HSV-1) infection and to elucidate the underlying mechanisms. Results: We found the expression of TRIM32 was increased after infected with HSV-1 both in murine corneas and cultured human epithelial (HCE) cells. Furthermore, knockdown of the expression of TRIM32 significantly aggravated HSV-1 induced herpetic stromal keratitis (HSK) in mice and promoted the replication of HSV-1 in cultured HCE cells. We also observed that silencing of TRIM32 resulted in the decreased expression of IFN- $\beta$ and suppressed activation of interferon regulatory factor 3 (IRF3) both in vivo and in vitro. Finally, we found TRIM32 positively regulate IFN- $\beta$ production in corneal epithelial cells through promoting K63-linked polyubiquitination of stimulator of interferon genes (STING). Conclusion: In conclusion, our data suggested that TRIM32 as a crucial positive regulator of HSV-1 induced IFN- $\beta$ production in corneal epithelial cells, and it played a predominant role in clearing HSV-1 from the cornea.

\section{Introduction}

Herpes simplex virus type 1 (HSV-1) is a prevalent viral pathogen of humans and a variety of animal species [1]. Most common clinical manifestations of HSV-1 infections are oral, genital and ocular lesions [2]. Ocular infection with HSV-1 initiated in epithelial and stromal layers always leads to a chronic inflammatory herpes simplex keratitis lesion, resulting mononuclear lymphocytes and neutrophils infiltration and pro-inflammatory cytokines production. Inflammatory responses could promote the elimination of the virus,

Hao Cui and Ying Liu contributed equally to this work. 
nonetheless, if uncontrolled, it can also be destructive to the host cornea, leading to tissue damage and loss of vision [3-5].

Type 1 interferons, including interferon- $\alpha$ (IFN- $\alpha$ ) and IFN- $\beta$, play pivotal roles in defense against the infection of both RNA and DNA virus [6,7]. During infection of DNA virus such as HSV-1, multiple intracellular DNA sensors contribute to the recognition of DNA nucleic acid, leading to the initiation of antiviral responses and interferon production [8-10]. Stimulator of interferon genes (STING), an endoplasmic reticulum (ER) resident transmembrane protein, has recently been identified as an essential controller of host defense against of microbial and viral cytosolic DNA, and it was found that STING is crucial for the intracellular DNA-mediated type 1 IFN production in immune cells and fibroblasts [11-14]. It has also been shown that STING is a central component of host resistance to HSV-1 in the cornea, and the presence of STING was crucial for sustained control of HSV-1 replication in the corneal epithelium and resistance to viral neuroinvasion $[15,16]$, but the precise mechanisms of the regulation of STING in response to HSV-1 infection in corneal epithelial cells still remain unknown.

The tripartite motif (TRIM) proteins constitute a family of over 70 distinct protein members in humans, and are involved in a variety of cellular functions including cell growth, differentiation, apoptosis, carcinogenesis and signal transduction [17-19]. Because of the conserved RING-domain, most TRIM proteins mainly act as E3 ubiquitin ligase to regulate the activity of substrates. In innate immunity, diverse TRIM proteins have been identified as essential regulators of antiviral responses, promoting lysine 48 (K48) or K63-linked polyubiquitination of various critical proteins, eventually leading to the degradation or activation of these substrate proteins [20-23].

In this study, we investigated the function of TRIM32 in response to HSV-1 infection in corneal epithelial cells in order to elucidate the underlying mechanisms. We found the expression of TRIM32 was upregulated upon HSV-1 challenge in mouse corneas and cultured HCE cells. Knockdown of the expression of TRIM32 significantly aggravated HSV-1 induced herpetic stromal keratitis (HSK) in mice, decreased IFN- $\beta$ expression and suppressed interferon regulatory factor 3 (IRF3) activation. Furthermore, we found TRIM32 positively regulate IFN- $\beta$ production in corneal epithelial cells through promoting K63linked polyubiquitination of STING. In conclusion, our data suggested that TRIM32 function as a positive regulator of STING in response to HSV-1 infection in mouse corneal epithelial cells, and it played a predominant role in clearing HSV-1 from the cornea.

\section{Materials and Methods}

\section{Mice, cells and virus}

8-week-old female C57BL/6J mice were obtained from Joint Ventures Sipper BK Experimental Animal (Shanghai, China). All animals were handled in accordance with the ARVO Statement for the Use of Animals in Ophthalmic and Vision Research. The human corneal epithelial (HCE) cell line (RCB1834 HCE-T) was obtained from Dr. Kozaburo Hayashi (National Eye Institute, Bethesda, MD, USA). The cells were grown in Minimal Essential Media (MEM; Gibco-Invitrogen Corp., Rockville, MD) and supplemented with penicillin, streptomycin, and $10 \%$ fetal bovine serum (FBS). The cells were incubated at $37^{\circ} \mathrm{C}$ in a $5 \% \mathrm{CO}_{2}-95 \%$ air incubator. Experiments were performed when cells were at 80\%-90\% confluence. HSV-1 strain RE was propagated in Vero cell monolayers (number CCL81; ATCC, Manassas, VA). Infected Vero cells were harvested, titrated, and stored in aliquots at $-80^{\circ} \mathrm{C}$ until used.

\section{Corneal HSV-1 Infection and Scoring}

The experiment was performed as previously described [24]. Corneal infections of mice were performed under ether anesthesia. The mice were lightly scarified on their corneas with a 27-gauge needle, and a 3- $\mu$ l drop containing $10^{4}$ plaque-forming units (PFU) HSV-1 RE was gently applied to one eye. Mice were scored for HSK severity by slit lamp biomicroscopy on each observation day. HSK severity was scored as fol- 


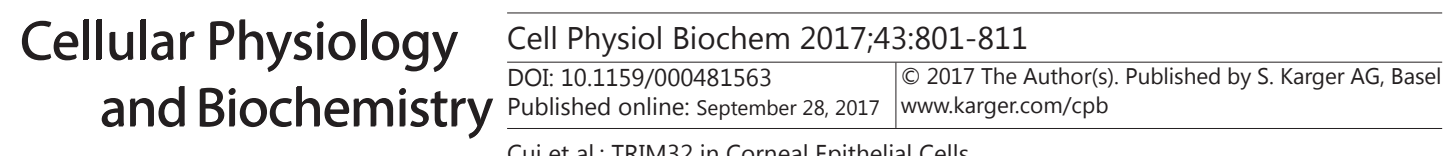

lows: 0 , normal cornea; 1, mild corneal haze; 2, moderate corneal opacity; 3 , severe corneal opacity; 4 , opaque cornea and ulcer; and 5 , corneal rupture. Corneal angiogenesis was scored by measuring the length of neovessels [25], stained with CD31 using deconvulted microscopy. Briefly, the CD31 stained corneal tissue specimens were divided into four quadrants. The length of the longest neovessel in each quadrant was graded between 0

Table 1. Primers used in the study

\begin{tabular}{lll}
\hline Number & Gene & Primer sequence \\
\hline 1 & TRIM32 & Forward 5'- AAGAAGATGGGGGCGAAAGG -3' \\
& mus musculus & Reverse 5'- GGCCCTCTCGAATAAGGACG -3' \\
& TRIM32 & Forward 5'- CCCGGACAGTTAACGTGGAA -3' \\
2 & homo spaiens & Reverse 5'- CGGGCTCATGTCCATCTCTC -3' \\
& IFN- $\beta$ & Forward 5'- ATGAGTGGTGGTTGCAGGC -3' \\
3 & mus musculus & Reverse 5'- TGACCTTTCAAATGCAGTAG -3' \\
& IFN- $\beta$ & Forward 5'- CAACAAGTGTCTCCTCCAAAT -3' \\
4 & homo spaiens & Reverse 5'- TCTCCTCAGGGATGTCAAAG -3' \\
& HSV-1 DNA & Forward 5'- CATCACCGACCCGGAGAGGGA - 3' \\
5 & polymerase & Reverse 5'- TCCACCACCCTGTTGCTGTA - 3' \\
& GAPDH & Forward 5'- ACCACAGTCCATGCCATCAC - 3' \\
6 & & Reverse 5'- TCCACCACCCTGTTGCTGTA - 3' \\
\hline
\end{tabular}
(no neovessel) and 4 (neovessel in the corneal center) in increments of about $0.4 \mathrm{~mm}$ (the radius of the cornea is about $1.5 \mathrm{~mm}$ ). The angiogenesis score for each cornea (ranging from 0 to 16) was the sum of the four quadrants. Virus titer in corneas was examined by plaque assay, tissues collected from euthanized animals for standard plaque assays were homogenized, and supernatants were tittered on confluent CCL-81 Vero cell (ATCC, Manassas, VA) monolayers in microtiter plates as described previously [26].

RNA Isolation and Quantitative Reverse Transcriptase PCR analysis

Total RNA was extracted with TRIzol reagent according to the manufacturer's instructions. A LightCycler (ABI PRISM 7000; Applied Biosciences) and a SYBR RT-PCR kit (Takara Biotechnology, Dalian, China) were used for quantitative reverse transcriptase PCR (qRT-PCR) analysis. The $2^{\wedge-\Delta \Delta C T}$ method was used to evaluate the relative quantities of each amplified product in the samples, to ensure equivalent loading and amplification, all products were normalized to GAPDH transcript as an internal control. Primer sequences used in quantitative RT-PCR are summarized in Table 1.

Lentivirus preparation and infection

Control-shRNA and TRIM32-shRNA Lentivirus were constructed by MDL technology company (MDL Biotech, Beijing, China). A customized lentiviral shRNA targeting TRIM32 was cloned into lentivirus vector (Invitrogen, BLOCK-iT ${ }^{\mathrm{TM}}$ Lentiviral RNAi Expression System, K4944-00). shRNA lentivirus was packaged and tittered in 93T cells according to the manufacturer's protocol. The enriched lentivirus particle (multiplicity of infection, MOI, 50) was used for cell lines infection in the presence of $5 \mu \mathrm{g} / \mathrm{ml}$ of polybrene (Sigma-Aldrich, St. Louis, USA). Lentivirus were subconjunctivally injected into the left eye of C57BL/6 J mice ( $5 \mu \mathrm{l} /$ mouse at a viral titer of $1 \times 10^{8}$ ) once a week for three times before ocular infection.

Western blot and ubiquitination assay

The corneas and HCE cells were lysed using RIPA buffer (MDL biotech, Beijing, China), and total protein in the supernatants was quantified using a BCA protein assay kit (Thermo Scientific, Waltham, MA). Western blots analysis and ubiquitination assay were performed as previously described [27]. The antibodies against TRIM32, STING, K63-linked ubiquitin (Abcam, Cambridge, USA), total-IRF3, phospho-IRF3 (Cell Signaling Technology Inc, Beverly, USA), tetherin (ThermoFisher Scientific, Shanghai, China), and GAPDH (Santa Cruz Biotechnology, Santa Cruz, USA) were used to probe the western blots. 


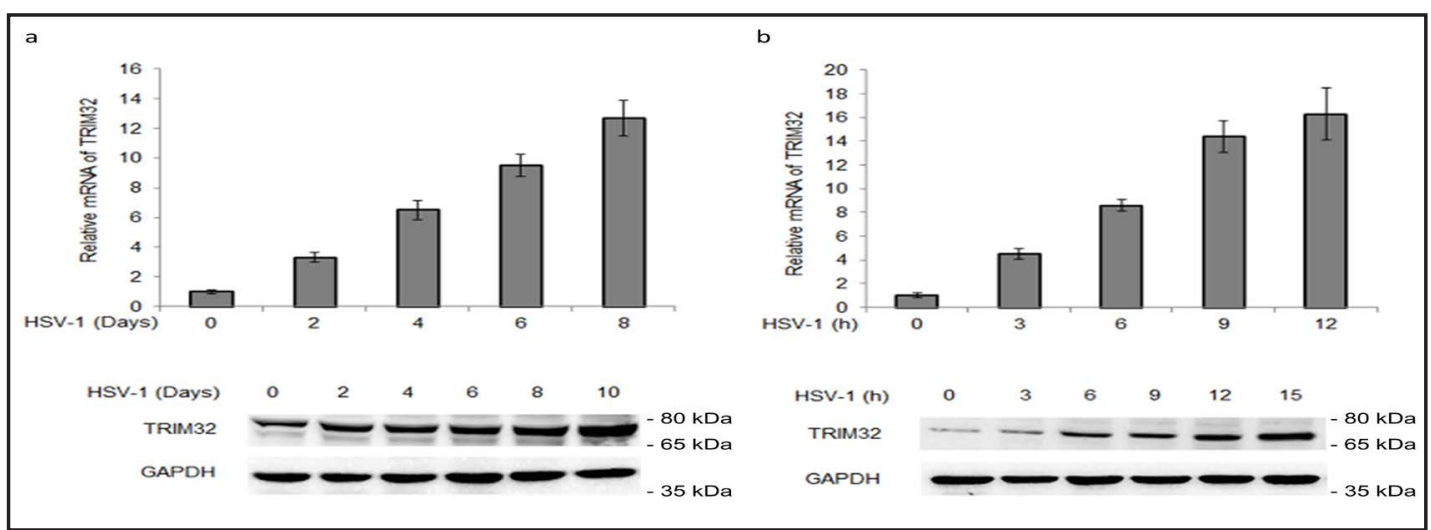

Fig. 1. TRIM32 expression was increased in mouse corneas and in vitro cultured HCE cells after HSV-1 infection. (a) Messenger RNA (mRNA) level (upper) and protein level (lower) of TRIM32 in mouse corneas after infection of HSV-1 for indicated time. (b) Messenger RNA (mRNA) level (upper) and protein level (lower) of TRIM32 in human corneal epithelial (HCE) cells after infection of HSV-1 for indicated time. The data are representative of three independent experiments (mean \pm SD).

ELISA

Cell supernatant of control-shRNA or TRIM32-shRNA lentivirus transduced HCE cells were collected 24 hours after HSV- 1 infection. Protein levels of TNF- $\alpha$, IL- 6 and IL- $1 \beta$ were measured using TNF- $\alpha$, IL- 6 and IL-1 $\beta$ ELISA kit (R\&D Systems, Minneapolis, USA) according to the manufacturer's instructions.

Dual-luciferase reporter gene assays

Luciferase activity of promoter was evaluated by Dual-Luciferase Reporter Assay System (Promega). Control-shRNA or TRIM32-shRNA lentivirus transduced HCE cells were transfected with luciferase reporter plasmids for IRF3 (Agilent, Santa Clara, CA); phRL-TK plasmid were co-transfected into the cells as internal control. 24 hours post-transduction with HSV luciferase activity was detected on a spectraMax M5 reader (Molecular Devices, California, USA).

\section{Statistical analysis}

All data were presented as mean \pm standard deviation (SD) of three experiments. Statistical significances were determined with the two-tailed Student $t$ test, with a p value less than 0.05 considered statistically significant.

\section{Results}

TRIM32 expression was increased in mouse corneas and in vitro cultured HCE cells after HSV-1 infection

To investigate the function of TRIM32 in HSV-1 infected mouse corneas, we first examined the expression of TRIM32 mRNA and protein after HSV-1 infection in corneas. As shown in Fig. 1(a), both mRNA and protein levels of TRIM32 were consistently expressed in the normal mouse corneas and gradually increased after HSV-1 infection. Corneal epithelial cells constitute the first line of defense against microbial pathogens such as virus, and they could also produce IFNs to enhance the antiviral activity of residential cells of the cornea [24]. Therefore we also examined the expression of TRIM32 in cultured HCE cells upon HSV-1 challenge. Consistent with the results in mouse corneas, in cultured HCE cells, we observed that TRIM32 expression was also upregulated at both mRNA and protein levels (Fig. 1(b)). Taken together, these results indicated that TRIM32 expression was induced by HSV-1 infection in corneal epithelial cells.

\section{KARGER}




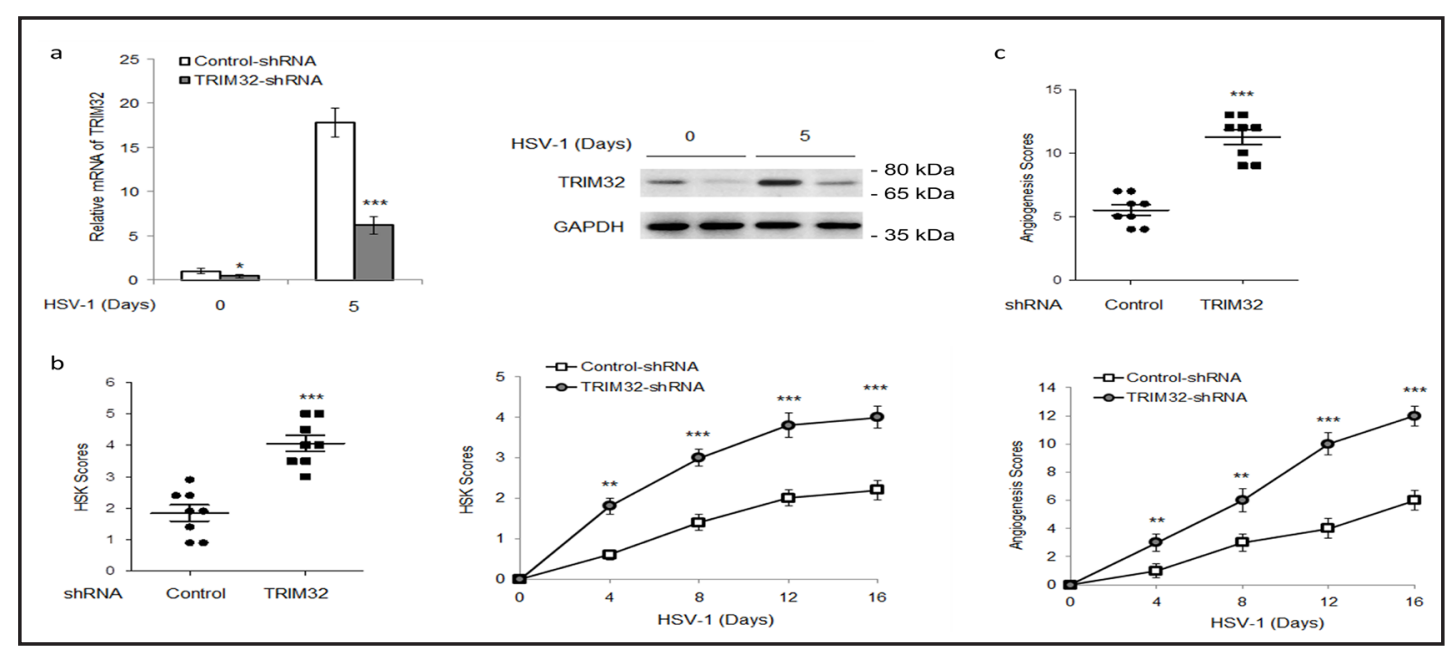

Fig. 2. Silencing of TRIM32 significantly aggravated HSV-1 induced herpetic stromal keratitis in mice. (a) TRIM32 mRNA level (left) and protein level (right) were examined in control-shRNA lentivirus or TRIM32-shRNA lentivirus treated mouse corneas at 5 days after HSV-1 infection to confirm the efficiency of knockdown expression of TRIM32. (b) HSK scores of day 16 (left) and average HSK scores from day 0-16 (right) after HSV-1 infection were calculated from control-shRNA lentivirus or TRIM32-shRNA lentivirus treated mice, 8 mice/time point. (c) Angiogenesis scores of day 16 (left) and average angiogenesis scores from day 0-16 (right) after HSV-1 infection were calculated from control-shRNA lentivirus or TRIM32-shRNA lentivirus treated mice, 8 mice/time point. The data are representative of three independent experiments (mean $\pm \mathrm{SD}$ ). ${ }^{* *} \mathrm{p}<0.01,{ }^{* * *} \mathrm{p}<0.001$ versus respective control group.

\section{Silencing of TRIM32 significantly aggravated HSV-1 induced herpetic stromal keratitis in} mice

Herpetic stromal keratitis (HSK) is the most common result from corneal infection with HSV-1, it causes more cases of monocular blindness than any other infectious eye disease in the world [28]. In order to determine the role of TRIM32 in HSV-1 induced corneal disease, C57BL/6 mice were subconjunctivally injected with control-shRNA lentivirus or TRIM32shRNA lentivirus, followed by HSV-1 infection to induce HSK experimental model. As shown in Fig. 2(a), both mRNA and protein levels of TRIM32 were decreased after challenged with TRIM32-shRNA lentivirus. Most important, HSK score, which reflected the severity of keratitis, was significantly increased in TRIM32-shRNA lentivirus treated corneas compared to the control-shRNA lentivirus treated corneas (Fig. 2(b)). Consistent with the HSK score results, the angiogenesis score, which is another important index in the evaluation of HSK development, was also significantly elevated in the TRIM32-shRNA lentivirus treated group (Fig. 2(c)). Taken together, these data indicated that silencing of TRIM32 significantly aggravated HSV-1 induced herpetic stromal keratitis in mice.

Knockdown of the expression of TRIM32 promoted the replication of HSV-1.x

Viral replication is an initial factor to induce recurrent HSK. To investigate the function of TRIM32 in HSV-1 replication, we used mouse corneas or cultured HCE cells treated with control-shRNA or TRIM32-shRNA lentivirus (Fig. 3(a)) followed by the HSV-1 infection, and we observed that the HSV-1 titer (Fig. 3(b)) and relative mRNA level of HSV-1 DNA polymerase (Fig. 3(c)) were all significantly increased after silencing of TRIM32 expression. These data suggested that TRIM32 can potentially inhibit replication of HSV-1 in corneal epithelial cells.

Knockdown of TRIM32 expression inhibited IFN- $\beta$ expression after HSV-1 infection

To explore the mechanism by which TRIM32 inhibited the replication of HSV-1, we first examined the production of type 1 IFN such as IFN- $\beta$ in lentivirus treated corneas and 
a

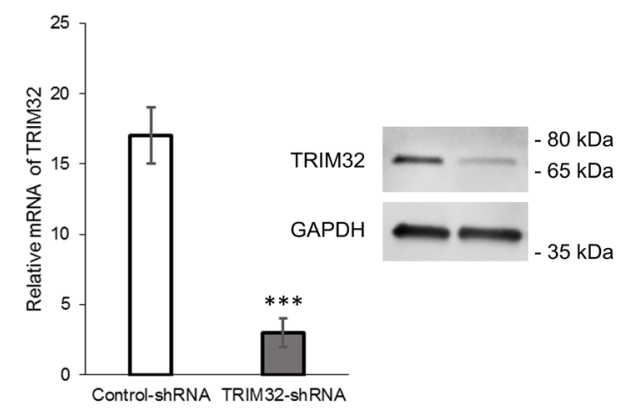

b

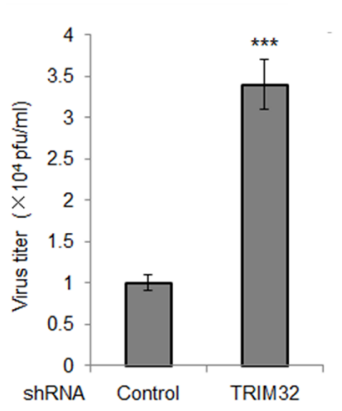

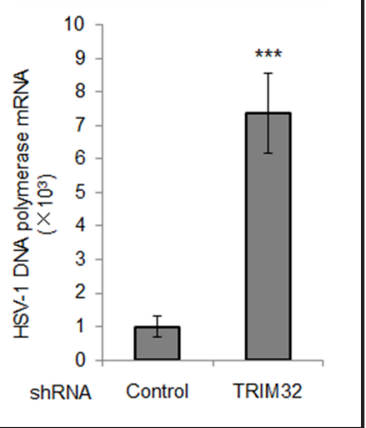

Fig. 3. Knockdown of the expression of TRIM32 promoted the replication of HSV-1. (a) TRIM32 mRNA level (left) and protein level (right) were examined in control-shRNA lentivirus or TRIM32-shRNA lentivirus treated HCE cells to confirm the efficiency of knockdown expression of TRIM32. (b) Plaque assay was performed to calculate virus titer in mouse corneas treated with control-shRNA lentivirus or TRIM32-shRNA lentivirus followed by HSV-1 infection for 5 days. (c) In HCE cells were pre-treated with control-shRNA lentivirus or TRIM32-shRNA lentivirus, relative levels of HSV-1 DNA polymerase mRNA was examined by qPCR after infected with HSV-1 for 12 hours. The data are representative of three independent experiments (mean $\pm \mathrm{SD}$ ). ${ }^{* * *} \mathrm{p}<0.001$ versus respective control group.

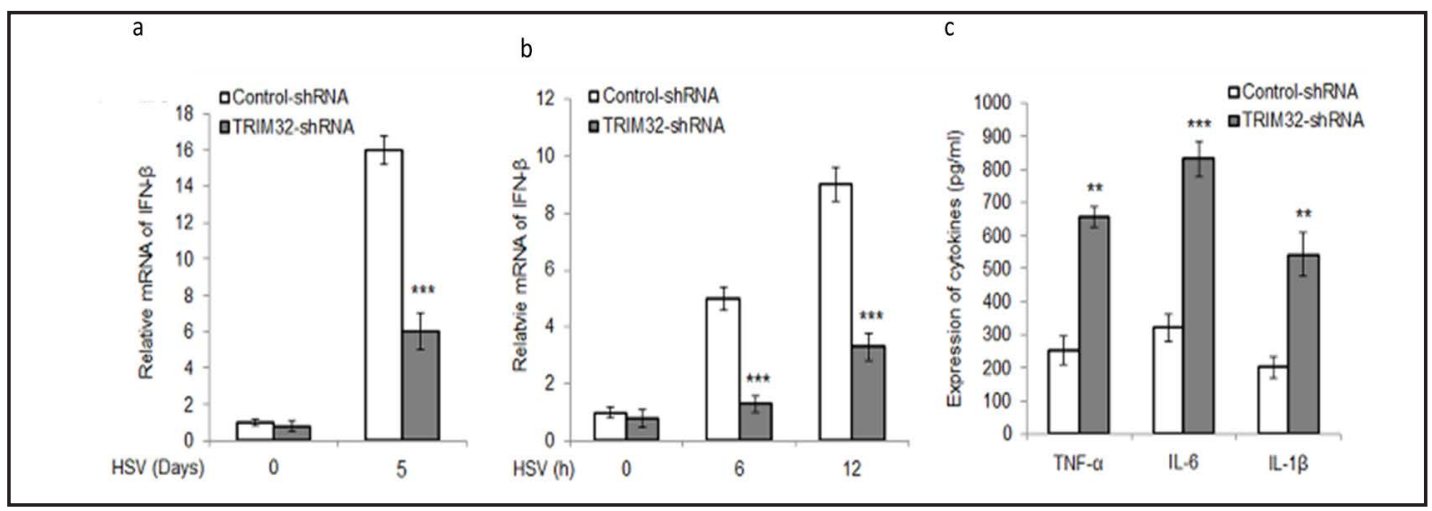

Fig. 4. Knockdown of TRIM32 expression inhibited IFN- $\beta$ expression after HSV-1 infection. (a) qRT-PCR assay was performed to examine the mRNA expression level of IFN- $\beta$ in mouse corneas infected with HSV-1 for indicated time. (b) qRT-PCR assay was performed to examine the mRNA expression level of IFN- $\beta$ in HCE cells infected with HSV-1 for indicated time. (c) Protein levels of secreted pro-inflammatory cytokines TNF- $\alpha$, IL- 6 and IL-1 $\beta$ in HCE cells were determined by ELISA. The data are representative of three independent experiments (mean $\pm \mathrm{SD}$ ). ${ }^{* *} \mathrm{p}<0.01,{ }^{* * *} \mathrm{p}<0.001$ versus respective control group.

HCE cells after HSV-1 infection. As shown in Fig. 4(a), the relative mRNA level of IFN- $\beta$ was significantly decreased in TRIM32-shRNA lentivirus treated corneas after HSV-1 infection. Consistent with this result, we found in HCE cells, the mRNA level of IFN- $\beta$ was also decreased after TRIM32-shRNA lentivirus treatment followed by HSV-1 infection (Fig. 4(b)). Furthermore, on evaluating the production of pro-inflammatory cytokines such as TNF- $\alpha$, IL-6, IL-1 $\beta$, we found the secreted pro-inflammatory cytokines were all increased in TRIM32shRNA lentivirus treated HCE cells (Fig. 4(c)). Taken together, these findings suggested that TRIM32 is a positive regulator of IFN- $\beta$ production but can also inhibit pro-inflammatory cytokines expression in corneal epithelial cells. 


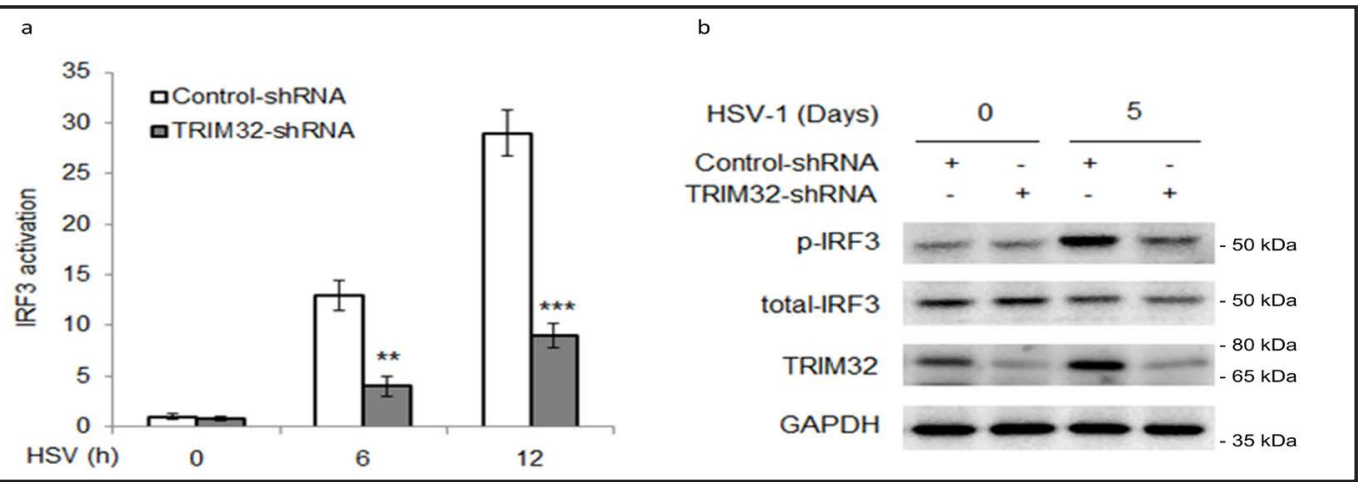

Fig. 5. Silencing of TRIM32 inhibited the activation of IRF3 in HCE cells and corneas. (a) HCE cells were treated with control-shRNA lentivirus or TRIM32-shRNA lentivirus, followed by transfection with IRF3 reporter plasmid together with phRL-TK plasmid (internal control). 24 hours post-transfection, the cells were infected with HSV-1 for indicated time, and the activation of IRF3 promoter was measured by DualLuciferase reporter gene assay. (b) Phosphorylation of IRF3 was examined by western blot assay in controlshRNA lentivirus or TRM32-shRNA lentivirus treated mouse corneas at indicated time after PA infection. The data are representative of three independent experiments (mean \pm SD). ${ }^{* *} \mathrm{p}<0.01,{ }^{* * *} \mathrm{p}<0.001$ versus respective control group.

Silencing of TRIM32 inhibited the activation of IRF3 in HCE cells and corneas

IRF3 is the main transcription factor involved in IFN- $\beta$ production [29]. In order to detect whether TRIM32 regulate IFN- $\beta$ production through regulation of IRF3 activation, we detected the activation of IRF3 by dual-luciferase reporter assay. As shown in Fig. 5(a), the activation of IRF3 was significantly inhibited in TRIM32-shRNA lentivirus treated HCE cells compared to the control groups. IRF3 activation requires the phosphorylation of conserved serine and threonine residues at the C-terminal region [29]. We observed that the phosphorylation of IRF3 was increased by HSV-1 infection, but in TRIM32-shRNA lentivirus treated corneas, the level of phosphorylation was significantly attenuated (Fig. 5(b)). Taken together, these data indicated that TRIM32 promoted the activation of IRF3 in response to the HSV-1 infection in HCE cells and corneas.

Knockdown of the expression of TRIM32 inhibited STING-induced IFN- $\beta$ production, decreased K63-linked polyubiquitination of STING, and increased expression of the viral restriction factor tetherin

STING was reported as the central regulator of host defense against of microbial and viral cytosolic DNA [14]. Previous research showed that TRIM32 modulates type 1 interferon production and cellular antiviral response by targeting STING protein for K63linked ubiquitination in 293T cells [27], thus we asked if TRIM32 regulate IRF3 activation through STING protein. Indeed, we found STING-induced activation of IFN- $\beta$ was attenuated by treatment of TRIM32-shRNA lentivirus in HCE cells (Fig. 6(a)). In addition, we examined the K63-linked polyubiquitination of STING in corneas after HSV-1 infection, and we found the K63-linked polyubiquitination of STING was decreased in TRIM32-shRNA lentivirus treated mouse corneas (Fig. 6 (b, c)). It has been previously identified that STING and STING induction of tetherin are both substantial anti-viral mechanism that contributes to host defense against HSV-1 infection in the cornea [16]. Hence, we next determined the effect of TRIM32-shRNA lentivirus on tetherin (also known as CD137, or BST-2) in the corneal epithelium. Tetherin expression was significantly downregulated in TRIM32-shRNA lentivirus treated mouse corneas, confirming that STING'S action is indeed mediated by tetherin expression [16]. Taken together, these results indicated that TRIM32 promote K63linked polyubiquitination of STING, leading to the activation of IRF3, increased production 


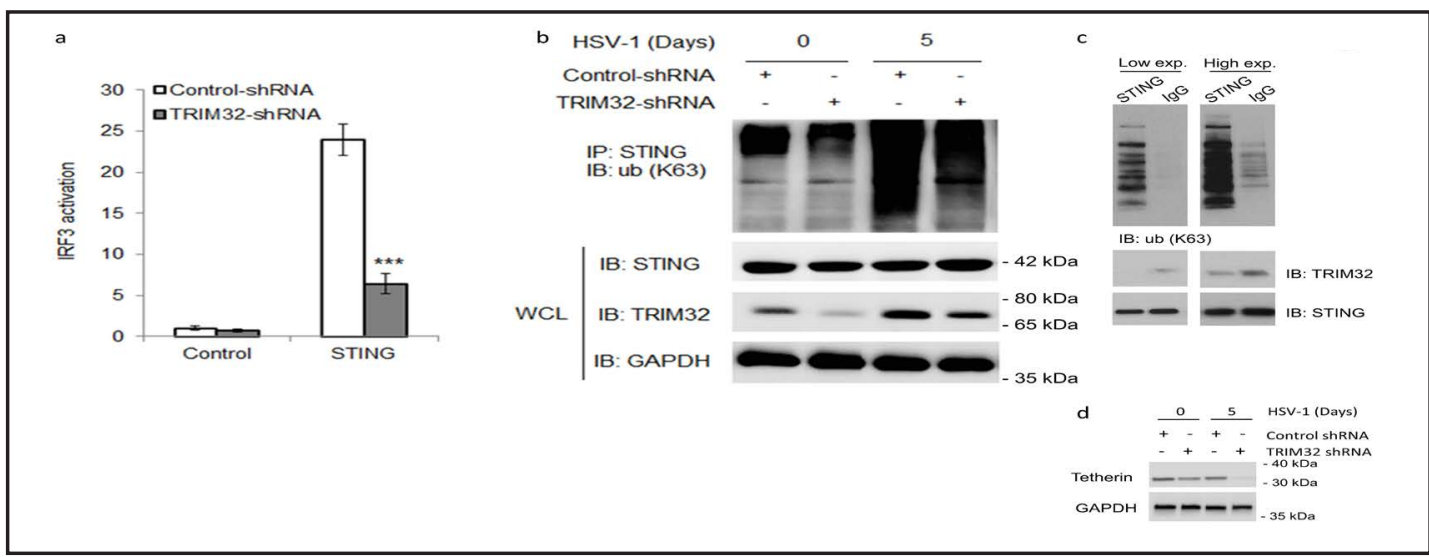

Fig. 6. Knockdown of the expression of TRIM32 inhibited STING-induced IFN- $\beta$ production and decreased K63-linked polyubiquitination of STING. (a) HCE cells were treated with control-shRNA lentivirus or TRIM32-shRNA lentivirus, followed by transfection with IRF3 reporter plasmid, phRL-TK plasmid, together with control or STING expression plasmid. 24 hours post-transfection, the activation of IRF3 promoter was measured by Dual-Luciferase reporter gene assay. (b, c) Western blot analysis of K63-linked polyubiquitination level of STING in control-shRNA lentivirus or TRIM32-shRNA lentivirus treated mouse corneas at indicated time after PA infection. Comparison of immunoprecipitated product with anti-STING or IgG antibody in 'c' confirmed that the ubiquitination smear observed in 'b' is that of STING. (d) Western blot analysis of STING target tetherin in control-shRNA lentivirus or TRIM32-shRNA lentivirus treated mouse corneas at indicated time after PA infection. The data are representative of three independent experiments (mean $\pm \mathrm{SD}$ ). ${ }^{* * *} \mathrm{p}<0.001$ versus respective control group.

of IFN- $\beta$, and efficient clearance of HSV-1 in corneal epithelial cells, therefore inhibiting the replication of HSV-1, resulting in the remission of HSK.

\section{Discussion}

We thus demonstrated that nsmmmmthe expression and function of TRIM32 in HSV-1 infected corneal epithelial cells and mouse corneas. To the best of our knowledge, this study is the first publication showing the relationship between TRIM32 and HSV-1-induced eye disease.

Previous studies have shown that TRIM32 could promote K48-linked polyubiquitination of various substrates to affect different physiological process and disease progression [3035]. As a supplement, our current study demonstrated the essential function of TRIM32 in HSV-1 infected corneal epithelial cells and corneas, and provided the evidence that TRIM32 delayed HSK progression through promoting K63-linked polyubiquitination of STING.

Herpes Simplex Virus type I (HSV-1) is a double strand DNA virus, its survival is dependent on its ability to exploit host cell machinery for replication, and intrinsic cellular defenses mainly contribute to the limitation of viral replication [36]. HSV-1 keratitis remains a leading cause of corneal morbidity; how to effectively eliminate the virus without causing a strong inflammatory response, has great significances for the treatment of HSV-1 keratitis. Previous study showed that expression of endothelial roundabout receptor 4 (Robo4) was increased after HSV-1 infection and activation of Robo4 reduces the severity of HSV-1induced keratitis [37]. Shen et al. reported that HSV-1 infection of the cornea induces CXCL10 expression in epithelial cells and absence of CXCL10 aggravates HSK in mice [38]. In the present study, we found the expression of TRIM32 was induced by HSV-1 infection in HCE cells and mouse corneas, and silencing of TRIM32 obviously aggravated HSK progression and virus replication. 
Innate immunity is crucial for the host to defense against invading pathogens. The initiation of the innate immune response depends on the recognition of pathogen associated molecular patterns (PAMPs) by pattern-recognition receptors (PRRs) including Toll-like receptors (TLRs), RIG-I-like receptors (RLRs), NOD-like receptors (NLRs) and intracellular DNA sensors $[37,38]$. The discovery of STING is one of the most exciting discoveries in the study of innate immunity in recent years, and the pathway including cyclic GAMP synthase (cGAS), STING, TBK1 and IRF3 has been well studied [12, 38]. cGAS can bind to DNA from DNA pathogens such as HSV-1 in the cytoplasm and subsequently catalyze the synthesis of cyclic guanosine monophosphate-adenosine monophosphate (cGAMP) from GTP and ATP,. cGAMP subsequently bind to STING, leading to ubiquitination and dimerization of STING to activate downstream tank binding kinase (TBK1), TBK1 then phosphorylate IRF3, followed by translocation of IRF3 into nuclear to initiate IFNs transcription [39]. Different from K48-linked polyubiquitination mediated degradation of target substrates, K63linked polyubiquitination of STING is essential for the activation of STING. Previous study demonstrated that E3 ubiquitin ligase TRIM56 mediates K63-linked polyubiquitination of STING, which potentiates IFN- $\beta$ production [40]. TRIM32 was also reported to target STING for K63-linked polyubiquitination and downstream activation in 29T cells [27]. In this study, we found TRIM32 could also promote K63-linked polyubiquitination of STING in mouse corneas and corneal epithelial cells in response to HSV-1 infection, and TRIM32 played pivotal roles in the suppression of HSK and HSV-1 replication.

The development of HSK is not only related to the infection of HSV-1, but also related to the uncontrolled inflammatory responses [41]. Interestingly, we found TRIM32 could promote the expression of IFN- $\beta$ to eliminate virus i]In addition, TRIM32 was also observed to contribute to the inhibition of pro-inflammatory cytokines production, which indicated TRIM32 play another anti-inflammation function after HSV-1 infection in corneal epithelial cells, and TRIM32 may affect the development of HSK through two regulatory mechanisms. But since we also observed decreased IRF3 activation and suppression of IFN-beta post silencing of TRIM32, we think that the observed effects on HSV replication is a direct effect of TRIM32. It is likely that the increased pro-inflammatory cytokines further potentiates this mechanism, which we plan to dissect further in our future studies. In addition, the precise mechanisms by which TRIM32 regulates the production of pro-inflammatory cytokines need to be further pursued.

In conclusion, our current study provided the evidence that TRIM32 expression was induced by HSV-1 infection. Our results cumulatively indicate that TRIM32 promote K63linked polyubiquitination of STING, leading to the activation of IRF3, increased production of IFN- $\beta$, and efficient clearance of HSV-1 in corneal epithelial cells, thereby inhibiting the replication of HSV-1, and ultimately resulting in the remission of HSK. These results suggest that TRIM32 is a promising therapeutic target for the treatment of HSV-1 induced keratitis.

\section{Abbreviations}

TRIM32 (tripartite motif protein 32); HSV-1 (Herpes Simplex Virus type 1); HCE (human epithelial cells); HSK (herpetic stromal keratitis); IRF3 (interferon regulatory factor 3); STING (stimulator of interferon genes); IFN- $\alpha$ (including interferon- $\alpha$ ); ER (endoplasmic reticulum); TRIM (the tripartite motif); FBS (fetal bovine serum); PFU (plaque-forming units).

\section{Acknowledgements}

None. 


\section{Cellular Physiology Cell Physiol Biochem 2017;43:801-811 \begin{tabular}{ll|l} 
DOI: 10.1159/000481563 & $\begin{array}{l}\text { O 2017 The Author(s). Published by S. Karger AG, Basel } \\
\text { www.karger.com/cpb }\end{array}$ \\
\hline and Biochemistry Published online: September 28, 201 &
\end{tabular}}

Cui et al.: TRIM32 in Corneal Epithelial Cells

\section{Disclosure Statement}

The authors declare that there is no conflict of interests.

\section{References}

$>1$ Xu F, Schillinger JA, Sternberg MR, Johnson RE, Lee FK, Nahmias AJ, Markowitz LE: Seroprevalence and coinfection with herpes simplex virus type 1 and type 2 in the United States, 1988-1994 J Infect Dis 2002;185:1019-1024.

-2 Biswas PS, Rouse BT: Early events in HSV keratitis—setting the stage for a blinding disease. Microbes Infect 2005;7:799-810.

3 Bonini S, Rama P, Olzi D, Lambiase A: Neurotrophic keratitis. Eye (Lond) 2003;17:989-995.

4 Conrady CD, Zheng M, Fitzgerald KA., Liu C. Carr DJJ: Resistance to HSV-1 infection in the epithelium resides with the novel innate sensor, IFI-16 Mucosal Immunol 2012;5:173-183.

5 Inoue, Y: Immunological aspects of herpetic stromal keratitis. Semin Ophthalmol 2008; 23:221-227.

6 Stetson DB, Medzhitov R: Type I interferons in host defense. Immunity 2006;25:373-381.

7 Wang BX, Fish EN: The yin and yang of viruses and interferons. Trends Immunol 2012;33:190-197.

8 Arpaia N, Barton GM: Toll-like receptors: key players in antiviral immunity. Curr Opin Virol 2011;1:447-454.

-9 Luecke S, Paludan SR: Chapter Two-Innate Recognition of Alphaherpesvirus DNA. Adv Virus Res 2015;92: 63-100.

10 Barber GN: Cytoplasmic DNA innate immune pathways. Immunol Rev 2011;243:99-108.

11 Ishikawa H, Barber GN: STING is an endoplasmic reticulum adaptor that facilitates innate immune signalling. Nature 2008;455:674-678.

12 Ishikawa H, Ma Z, Barber GN: STING regulates intracellular DNA-mediated, type I interferon-dependent innate immunity. Nature 2009;461:788-792.

13 Abe T, Harashima A, Xia T, Konno H, Konno K, Morales A, Ahn J, Gutman D, Barber GN: STING recognition of cytoplasmic DNA instigates cellular defense. Mol Cell 2013;50: 5-15.

14 Zhong B, Yang Y, Li S, Wang YY, Li Y, Diao F, Lei C, He X, Zhang L, Tien P, Shu HB: The adaptor protein MITA links virus-sensing receptors to IRF3 transcription factor activation. Immunity 2008;29:538-550.

15 Parker ZM, Murphy AA, Leib DA: Role of the DNA sensor STING in protection from lethal infection following corneal and intracerebral challenge with herpes simplex virus 1. J Virol 2015;89:11080-11091.

16 Royer DJ, Carr DJ: A STING-dependent innate-sensing pathway mediates resistance to corneal HSV-1 infection via upregulation of the antiviral effector tetherin. Mucosal Immunol 2016;9:1065-1075.

17 Lascano J, Uchil PD, Mothes W, Luban J: TRIM5 Retroviral Restriction Activity Correlates with the Ability To Induce Innate Immune Signaling. J Virol 2016;90:308-316.

-18 Wang Y, He D, Yang L, Wen B, Dai J, Zhang Q Kang J, He W, Ding Q, He D: TRIM26 functions as a novel tumor suppressor of hepatocellular carcinoma and its downregulation contributes to worse prognosis. Biochem Biophys Res Commun 2015;463:458-465.

19 Shibata M, Sato T, Nukiwa R, Ariga T, Hatakeyama S: TRIM45 negatively regulates NF- $\mathrm{kB}-\mathrm{mediated}$ transcription and suppresses cell proliferation. Biochem Biophys Res Commun 2012;423:104-109.

-20 Narayan K, Waggoner L, Pham ST, Hendricks GL, Waggoner SN, Conlon J, Wang JP, Fitzgerald KA, Kang J: TRIM13 is a negative regulator of MDA5-mediated type I interferon production. J Virol 2014;88:1074810757.

-21 Yan J, Li Q, Mao AP, Hu MM, Shu HB: TRIM4 modulates type I interferon induction and cellular antiviral response by targeting RIG-I for K63-linked ubiquitination. J Mol Cell Biol 2014;6:154-163.

22 Yang C, Zhao X, Sun D, Yang L, Chong C, Pan Y, Chi X, Gao Y, Wang M, Shi X, Sun H, Lv J, Gao Y, Zhong J, Niu J, Sun B: Interferon alpha (IFN\&agr;)-induced TRIM22 interrupts HCV replication by ubiquitinating NS5A. Cell Mol Immunol 2016;13:94-102.

-23 Fletcher AJ, Mallery DL, Watkinson RE, Dickson CF, James LC: Sequential ubiquitination and deubiquitination enzymes synchronize the dual sensor and effector functions of TRIM21. Proc Natl Acad Sci USA 2015;112:10014-10019. 


\section{Cellular Physiology Cell Physiol Biochem 2017;43:801-811 \begin{tabular}{l|l|l|l} 
DOI: 10.1159/000481563 & O 2017 The Author(s). Published by S. Karger AG, Basel \\
www.karger.com/cpb
\end{tabular} \\ Cui et al.: TRIM32 in Corneal Epithelial Cells}

24 Mulik S, Sharma S, Suryawanshi A, Veiga-Parga T, Reddy PB, Rajasagi NK, Rouse BT: Activation of endothelial roundabout receptor 4 reduces the severity of virus-induced keratitis. J Immunol 2011;186:7195-7204.

-25 Shen FH, Wang SW, Yeh TM, Tung YY, Hsu SM, Chen SH: Absence of CXCL10 aggravates herpes stromal keratitis with reduced primary neutrophil influx in mice. J Virol 2013;87:8502-8510.

-26 Royer DJ, Zheng M, Conrady CD, Carr DJ: Granulocytes in Ocular HSV-1 Infection: Opposing Roles of Mast Cells and NeutrophilsGranulocytes in Ocular HSV-1 Infection. Invest Ophthalmol Vis Sci 2015;56:3763-3775.

-27 Zhang J, Hu MM, Wang YY, Shu HB: TRIM32 protein modulates type I interferon induction and cellular antiviral response by targeting MITA/STING protein for K63-linked ubiquitination. J Biol Chem 2012; 287:28646-28655.

-28 Liesegang TJ: Herpes simplex virus epidemiology and ocular importance. Cornea 2001;20:1-13.

29 Wathelet MG, Lin CH, Parekh BS, Ronco LV, Howley PM, Maniatis T: Virus infection induces the assembly of coordinately activated transcription factors on the IFN- $\beta$ enhancer in vivo. Mol Cell 1998;1:507-518.

-30 Schoser BG, Frosk P, Engel AG, Klutzny U, Lochmüller H, Wrogemann K: Commonality of TRIM32 mutation in causing sarcotubular myopathy and LGMD2H. Ann Neurol 2005;57:591-595.

-31 Kudryashova E, Wu J, Havton LA, Spencer MJ: Deficiency of the E3 ubiquitin ligase TRIM32 in mice leads to a myopathy with a neurogenic component. Hum Mol Genet 2009;18:1353-1367.

\$2 Chiang AP, Beck JS, Yen HJ, Tayeh MK, Scheetz TE, Swiderski RE, Nishimura DY, Braun TA, Kim KY, Huang J, Elbedour K, Carmi R, Slusarski DC, Casavant TL, Stone EM, Sheffield VC: Homozygosity mapping with SNP arrays identifies TRIM32, an E3 ubiquitin ligase, as a Bardet-Biedl syndrome gene (BBS11). Proc Natl Acad Sci U S A 2006;103:6287-6292.

-33 Albor A, El-Hizawi S, Horn EJ, Laederich M, Frosk P, Wrogemann K, Kulesz-Martin M: The interaction of Piasy with Trim32, an E3-ubiquitin ligase mutated in limb-girdle muscular dystrophy type $2 \mathrm{H}$, promotes Piasy degradation and regulates UVB-induced keratinocyte apoptosis through NFKB. J Biol Chem 2006;281:2585025866.

-34 Saccone V, Palmieri M, Passamano L, Piluso G, Meroni G, Politano L, Nigro V: Mutations that impair interaction properties of TRIM32 associated with limb - girdle muscular dystrophy 2H. Hum Mutat 2008;29: 240-247.

-35 Locke M, Tinsley CL, Benson MA, Blake DJ: TRIM32 is an E3 ubiquitin ligase for dysbindin. Hum Mol Genet 2009;18:2344-2358.

-36 Siracusano G, Venuti A, Lombardo D, Mastino A, Esclatine A, Sciortino MT: Early activation of MyD88mediated autophagy sustains HSV-1 replication in human monocytic THP-1 cells. Sci Rep 2016;6:31302.

37 Akira S, Uematsu S, Takeuchi O:Pathogen recognition and innate immunity. Cell 2006;124:783-801.

38 Medzhitov R: Recognition of microorganisms and activation of the immune response. Nature 2007;449:819826.

-39 Ran Y, Shu HB, Wang YY: MITA/STING: A central and multifaceted mediator in innate immune response. Cytokine Growth Factor Rev 2014;25:631-639.

40 Tsuchida T, Zou J, Saitoh T, Kumar H, Abe T, Matsuura Y, Kawai T, Akira S: The ubiquitin ligase TRIM56 regulates innate immune responses to intracellular double-stranded DNA. Immunity 2010;33:765-776.

-41 Rowe AM, Leger AS, Jeon S, Dhaliwal DK, Knickelbein JE, Hendricks RL: Herpes keratitis. Prog Retin Eye Res 2013;32:88-101. 